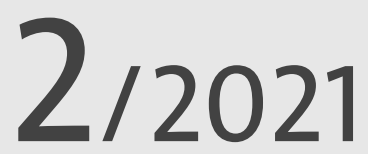

27. Jahrgang, Seiten 49-104

Herausgeber: Univ.-Prof. em. Dr. Heinrich Reinermann, Deutsche Universität für Verwaltungswissenschaften Speyer | Univ.-Prof. Dr. Veith Mehde, Mag.rer.publ., Leibniz Universität Hannover (geschäftsführend) | Prof. Dr. Tino Schuppan, Hochschule der Bundesagentur für Arbeit, Schwerin (geschäftsführend)

Beirat: Helmut Dedy, Geschäftsführendes Präsidialmitglied des Deutschen Städtetages, Köln | Dr. Hans Bernhard Beus, Staatssekretär a.D. im Bundesministerium der Finanzen, Berlin | Prof. Dr. Martin Brüggemeier, Hochschule für Technik und Wirtschaft, Berlin | Hans Jörg Duppré, Landrat, Präsident des Deutschen Landkreistages, Berlin | Univ.-Prof. Dr. Gisela Färber, Deutsche Universität für Verwaltungswissenschaften Speyer | Prof. Dr. Gerhard Hammerschmid, Hertie School of Governance GmbH, Berlin | Peter Heesen, Bundesvorsitzender des Deutschen Beamtenbundes, Bonn | Dr. Gerd Landsberg, Geschäftsführendes Präsidialmitglied des Deutschen Städte- und Gemeindebundes, Berlin | Prof. Dr. Andreas Lasar, Hochschule Osnabrück | Dr. Johannes Meier, Mitglied des Vorstands der Bertelsmann-Stiftung, Gütersloh | Univ.-Prof. Dr. Isabella Proeller, Universität Potsdam | Prof. Dr. Marga Pröhl, Generaldirektorin des European Institute of Public Administration (EIPA), Maastricht | Dr. Sebastian Saxe, Mitglied der Geschäftsleitung der Hamburg Port Authority Anstalt des öffentlichen Rechts, Hamburg | Univ.-Prof. Dr. Christina Schaefer, Helmut Schmidt Universität, Hamburg | Kay Scheller, Präsident des Bundesrechnungshofes, Bonn | Prof. Dr. Reto Steiner, ZHAW School of Management and Law, Winterthur | Prof. Dr. Arthur Winter, Donau-Universität Krems | Christian Zahn, Mitglied des Bundesvorstands der Vereinten Dienstleistungsgewerkschaft ver.di, Berlin

\title{
Verbreitung von Kirchgemeindefusionen in der Schweiz
}

\author{
Reto Steiner/Marcel Notter
}

Während die Literatur zu Fusionen von politischen Gemeinden sowohl im deutsch- wie auch im englischsprachigen Raum umfangreich ist, ist bei den Kirchgemeinden als öffentlich-rechtliche Körperschaften das Thema bislang nahezu unerforscht. Erst seit wenigen Jahren werden Zusammenschlüsse von Kirchgemeinden in der Schweiz in größerem Umfang vollzogen. Zum ersten Mal liegt gestützt auf eine Umfrage in den 26 Schweizer Kantonen eine Übersicht über die Fusionsaktivität von Kirchgemeinden in der Schweiz vor. Die Analyse zeigt, dass die Verbreitung kantonal unterschiedlich intensiv ist und die ersten Erfahrungen tendenziell positiv ausfallen.

\section{Einleitung}

Die Religion, um es mit den Worten von Jacob Burckhardt auszudrücken, ist „eine der drei Potenzen der Weltgeschichte ne-

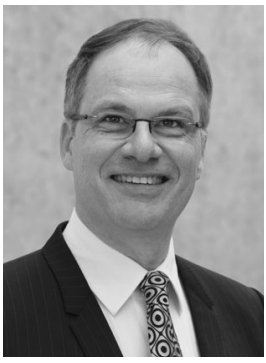

Prof. Dr. Reto Steiner Professor für öffentliches Management und Direktor der ZHAW Schoo of Management and Law in Winterthur (Schweiz). ben Kultur und Staat". ${ }^{1}$ Immer noch gehören 59,6 Prozent der ständigen Wohnbevölkerung in der Schweiz einer der beiden großen christlichen Konfessionen an

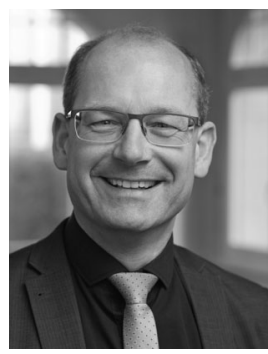

Marcel Notter

Executive MPA, ist Generalsekretär der Römisch-Katholischen Landeskirche des Kantons Aargau in Aarau (Schweiz).
- der evangelisch-reformierten oder der römisch-katholischen. Der Anteil an Konfessionslosen hat jedoch in den vergangenen Jahrzehnten deutlich zugenommen und nur noch ein Viertel der Bevölkerung besucht mehr als sechsmal pro Jahr einen Gottesdienst. ${ }^{2}$ Insbesondere die beiden großen Konfessionen, welche in der Regel als öffentlich-rechtlich anerkannte Körperschaften (Kirchgemeinden oder auch Kirchengemeinden genannt) organisiert sind, leiden an Mitgliederschwund und damit an sinkenden Finanzmitteln, der Schwierigkeit die Behördenämter zu besetzen und an einer teuren, aber weniger oft genutzten Infrastruktur. Um handlungsfähig zu bleiben, werden Fusionen von Kirchgemeinden seit einigen Jahren zu einem Thema, eine Entwicklung, welche bei den politischen Gemeinden bereits deutlich länger zu beobachten ist.

Burckhardt 1978, S. $39 \mathrm{ff}$.

Bundesamt für Statistik 2021. 
Der Beitrag stellt basierend auf einer empirischen Erhebung erstmal die Fusionsaktivitäten der Kirchgemeinden in der Schweiz dar und gibt einen Einblick in die Erfahrungen mit vollzogenen Fusionen. In einem ersten Teil des Beitrags wird die Stellung von Kirchgemeinden rechtlich und historisch eingeordnet. Im Gegensatz zu den politischen Gemeinden, welche es in den meisten Ländern gibt, ist das Konstrukt der Kirchgemeinde nur in wenigen Ländern verbreitet, so etwa in der Schweiz und in Deutschland. In einem zweiten Teil zeigt der Beitrag die Verbrei- tone haben die römisch-katholische, die evangelisch-reformierte und die kleinere christkatholische (Synonym: altkatholische) Kirche und vereinzelt die jüdischen Religionsgemeinschaften öffentlich-rechtlich anerkannt. ${ }^{6}$

Die Schweiz kennt ein Nebeneinander von Kantonen mit mehr oder weniger strenger Trennung von Staat und Kirche. Typisch für das Schweizer Konstrukt ist die Nähe von Staat und Kirche mit kirchenhoheitlichen Elementen und Zuständigkeiten. So sind das Recht auf Kirchen-

\section{"Trotz einer feststellbaren Entwicklung hin zu einer stärkeren Trennung von Staat und Kirche wird die Stellung der Kirchen als öffentlich-rechtliche Körperschaften in der Schweiz erst vereinzelt in Frage stellt."}

tung und erste Auswirkungen von Kirchgemeindefusionen in der Schweiz auf, bevor Schlussfolgerungen gezogen werden.

\section{Rechtliche Stellung von Kirchgemeinden}

Eine Analyse der rechtlichen Stellung von christlichen Kirchen im internationalen Vergleich zeigt, dass es unterschiedliche Modelle gibt: Während in Frankreich und den USA ein Trennungsmodell mit einer mehr oder weniger strikten Trennung von Staat und Kirche verbreitet ist, kennen andere Länder eine Staatskirche oder zumindest etablierte Großkirchen. $\mathrm{Zu}$ dieser Ländergruppe gehören beispielsweise Skandinavien, Großbritannien und Griechenland. Auch die Schweiz und Deutschland können dieser Gruppe zugeordnet werden. ${ }^{3}$

Die Schweizerische Bundesverfassung (BV) weist die Zuständigkeit für die Regelung der Beziehung zwischen Staat und Kirche den Kantonen zu. ${ }^{4}$ Die Kirchen haben ihre staatsrechtliche Grundlage somit im kantonalen Recht. ${ }^{5}$ Fast alle Kan- steuern und Leistungen des Staates an die Kirchen weit verbreitet. ${ }^{7}$

Das schweizerische System genießt trotz einer feststellbaren Entwicklung hin $\mathrm{zu}$ einer stärkeren Trennung von Staat und Kirche (so sind beispielsweise im Kanton Bern die Pfarrpersonen seit 2021 durch die Kirchen angestellt und nicht mehr durch den Staat) nach wie vor Unterstützung und die Stellung der Kirchen als öffentlich-rechtliche Körperschaften wird erst vereinzelt in Frage stellt. Als Vorteile werden etwa genannt:

- Die Rechtsverbindlichkeit von demokratischen Verfahren, die Rechtsstaatlichkeit, die Gewaltenteilung, die Gleichstellung von Mann und Frau und die finanzielle Transparenz sind im Zuständigkeitsbereich der staatskirchenrechtlichen Körperschaften garantiert.

- Dank des föderativen Staatsaufbaues und des hohen Stellenwertes des Subsidiaritätsprinzips sind die Kirchen dezentral aufgebaut.

- Die Inkulturation der kirchlichen Organisation in die direktdemokratischen und föderalistischen Staatsstrukturen und in die politische Kultur des Landes erhöht tendenziell den gesellschaftlichen Rückhalt.

- Das Einbringen von Erfahrungen und Kompetenzen in den Bereichen Verwaltung, Finanzen, Management und die Schaffung rechtlicher Regelungen könnte auf die Kirchen qualitätsfördernd wirken.

- Die Vereinbarkeit von Berufstätigkeit und Mitarbeit in staatskirchenrechtlichen Organen erlaubt es, unterschiedliche Kompetenzen und Lebenserfahrungen in das kirchliche Milizsystem einfließen zu lassen. ${ }^{8}$

In der reformierten Kirche ist das Konzept der Kirchgemeinde relativ einfach geregelt: Diese sind grundsätzlich für alle Aufgaben, welche in einer Kirchgemeinde anfallen, zuständig. Gewisse Rahmenbedingungen und Vorgaben (wie z.B. Formulierung der Anstellungsvoraussetzungen für Pfarrpersonen) können von den kantonalen Kirchen kommen, die ebenfalls öffentlich-rechtlich verfasst sind. Die Kantonskirchen sind wiederum als Verein in der Evangelischen Kirche Schweiz zusammengefasst, welche aber primär die Interessen der Reformierten auf nationaler Ebene wahrnimmt.

In der katholischen Kirche bleibt das Nebeneinander von römisch-katholischer Weltkirche mit dem dazugehörigen kanonischen Kirchenrecht und staatsrechtlichen Strukturen eine Herausforderung (duales System). Die Organe gliedern sich in zwei Bereiche mit jeweils unterschiedlichen Aufgaben. Neben den Organen des kirchlichen Rechts (Bistümer, Pfarreien), die sich in erster Linie um Fragen der Kirchenleitung, der Pastoral, Verkündigung und Sakramente kümmern, existieren die Organe des staatlichen Rechts (Kirchgemeinden und kantonale kirchliche Körperschaften), die um Verwaltungsangelegenheiten, Finanzen, Liegenschaften und

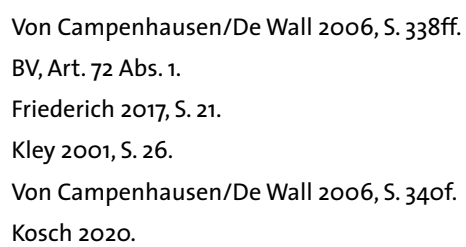




\begin{tabular}{|c|c|c|c|c|}
\hline & $\begin{array}{c}\text { Evangelisch- } \\
\text { reformiert }\end{array}$ & $\begin{array}{l}\text { Römisch- } \\
\text { katholisch }\end{array}$ & $\begin{array}{c}\text { Christ- } \\
\text { katholisch }\end{array}$ & $\begin{array}{l}\text { Jüdische } \\
\text { Gemeinde }\end{array}$ \\
\hline Aargau & $x$ & $x$ & $x$ & \\
\hline Appenzell-Ausserrhoden & $x$ & $x$ & & \\
\hline Appenzell-Innerrhoden & $x$ & $x$ & & \\
\hline Basel-Landschaft & $x$ & $x$ & $x$ & \\
\hline Basel-Stadt & $x$ & $x$ & $x$ & $x$ \\
\hline Bern & $x$ & $x$ & $x$ & $x$ \\
\hline Freiburg & $x$ & $x$ & & $x$ \\
\hline \multicolumn{5}{|l|}{ Genf } \\
\hline Glarus & $x$ & $x$ & & \\
\hline Graubünden & $x$ & $x$ & & \\
\hline Jura & $x$ & $x$ & & \\
\hline Luzern & $x$ & $x$ & $x$ & \\
\hline \multicolumn{5}{|l|}{ Neuenburg } \\
\hline Nidwalden & $x$ & $x$ & & \\
\hline Obwalden & $x$ & $x$ & & \\
\hline St. Gallen & $x$ & $x$ & $x$ & $x$ \\
\hline Schaffhausen & $x$ & $x$ & $x$ & \\
\hline Schwyz & $x$ & $x$ & & \\
\hline Solothurn & $x$ & $x$ & $x$ & \\
\hline Tessin & $x$ & $x$ & & \\
\hline Thurgau & $x$ & $x$ & & \\
\hline Uri & $x$ & $x$ & & \\
\hline Waadt & $x$ & $x$ & & \\
\hline Wallis & $x$ & $x$ & & \\
\hline Zug & $x$ & $x$ & & \\
\hline Zürich & $\mathrm{x}$ & $\mathrm{x}$ & $x$ & \\
\hline
\end{tabular}

Abb. 1: Öffentlich-rechtlich anerkannte Landeskirchen resp. Religionsgemeinschaften nach Kantonen

Immobilien besorgt sind. Für die vorgestellte Arbeit ist das duale System insofern relevant, als eine staatskirchenrechtlich verfasste Kirchgemeinde mehrere kirchlich verfasste Pfarreien umfassen kann. Folglich können zwei Kirchgemeinden fusionieren, ohne dass auch die Pfarreien den Zusammenschluss vollziehen.

Das mittelalterliche Kirchenrecht kannte die Kirchgemeinden als Rechtsträgerin nicht, sondern betrachtete sie als Pfarrei im Sinne einer Untereinheit des Bistums. Trotzdem bildeten sich im Spätmittelalter im Zusammenhang mit der Entstehung von städtischen und ländlichen Gemeinden auch genossenschaftlich handelnde Kirchverbände. Besonders in der Innerschweiz erwarben Gemeinden im 15. Jahrhundert Formen der Mitbestimmung bis zur Präsentation eines Pfarrers. Die
Reformation machte die Kirchgemeinden in verschiedenen Kantonen zum zentralen Element der kirchlichen Verfassung. ${ }^{9}$

Prägend waren das napoleonische Zeitalter und die Zeit des Kulturkampfes. In der ersten Hälfte des 19. Jahrhunderts erhielt die Schweiz ihre heutige Gestalt, indem sie sich vom Staatenbund zum Bundesstaat wandelte. Gleichzeitig führte die Säkularisierung der kirchlichen Güter dazu, dass der Staat in manchen Kantonen kirchliche Unterhaltskosten übernahm, die bis heute als „Staatsbeiträge“ entrichtet werden. Schließlich waren die liberalen Kräfte daran interessiert, den päpstlichen und klerikalen Einfluss zurückzubinden, was erstens durch staatliche Aufsicht über das Kirchenwesen, zweitens durch inzwischen aufgehobene konfessionelle Ausnahmeartikel in der
Bundesverfassung und drittens durch die Errichtung von kantonalen Landeskirchen bewerkstelligt wurde. ${ }^{10}$ Fluchtpunkt der meisten Entwicklungen wurde im 20. Jahrhundert die öffentlich-rechtliche Anerkennung der Glaubensgemeinschaften mit Zuteilung der Steuerhoheit unter der Voraussetzung von demokratischen Strukturen und von Verwaltungstransparenz. ${ }^{11}$

Die Ausnahme für diese Entwicklung bilden die Kantone Genf, Neuenburg, Wallis und Tessin. Genf und Neuenburg als so genannte Trennungskantone kennen keine obligatorischen Kirchensteuern, hier nehmen privatrechtliche Vereine auf kantonaler und lokaler Ebene die Aufgaben wahr, die in der Mehrheit der Kantone von den öffentlich-rechtlich anerkannten Körperschaften wahrgenommen werden. Im Wallis ist die Struktur eng mit den politischen Einwohnergemeinden verwoben, die für finanzielle Defizite von Kirchgemeinden aufkommen. Im Tessin wiederum bestehen keine eigenen staatskirchenrechtlichen Körperschaften. Sowohl im Wallis wie auch im Tessin existiert in der katholischen Kirche kein duales System.

Abbildung 1 zeigt, in welchen Kantonen die Kirchen öffentlich-rechtlich anerkannt sind und entsprechende Strukturen kennen. In den Kantonen mit öffentlichrechtlicher Anerkennung sind die lokalen Kirchgemeinden juristische Personen des öffentlichen Rechts. Die lokalen Kirchgemeinden schließen sich jeweils auf kantonaler Ebene zu einer eigenen Körperschaft zusammen, welche mit wenigen Ausnahmen wiederum öffentlich-rechtlich anerkannt ist. Mitglieder der (kantonalen) Landeskirchen sind die Kirchgemeinden.

\section{Quantitative und qualitative Erhebung}

Im Rahmen einer empirischen Untersuchung soll untersucht werden, ob es bei den Kirchgemeinden in den vergangenen Jahren analog zu den politischen Gemeinden in der Schweiz zu Gemeindezusammenschlüssen gekommen ist. Als Grund-

\footnotetext{
9 Kunz/Schlag 2017, S. 74 f

10 Kosch 2007, S. 9.

11 Stadler 2010
} 
gesamtheit werden alle öffentlich-rechtlich verfassten Kirchgemeinden der katholischen, der evangelisch-reformierten und der christkatholischen Kirchen der Schweiz gewählt.

Die 67 schriftlichen Fragebogen wurden an die Kantonalkirchen in der Deutschschweiz, in der Westschweiz sowie in der italienischsprachigen Schweiz versandt, welche die Situation in den lokalen Kirchgemeinden ihres Kantons kennen. Insgesamt sind 56 ausgefüllte Fragebögen eingegangen (Rücklaufquote: 83 Prozent). Die Befragung fokussierte auf die Erfassung aller umgesetzten und gescheiterten Fusionen von Kirchgemeinden zwischen 2010 und 2018.

Als Ergänzung zur schriftlichen Befragung wurden drei umgesetzte Kirchgemeindefusionen in den Kantonen Bern, Aargau und Zürich im Rahmen von Fallstudien vertieft analysiert. Dazu wurden problemzentrierte Interviews mit Kirchgemeindepräsidien, Mitgliedern der

Finanzkommission, Verwaltungen und Pfarrpersonen geführt. Es handelt sich dabei um die Kirchgemeinden Saanen und Gsteig (reformiert, Kanton Bern), Hornussen und Zeihen (römisch-katholisch, Kanton Aargau) sowie Dübendorf und Schwerzenbach (reformiert, Kanton Zürich). Die Fallstudien geben einen Einblick in die Auswirkungen realisierter Fusionsprojekte.

Sowohl bei der quantitativen wie auch bei der qualitativen Befragung wurde auf drei Analysedimensionen gesetzt: Als erstes wurden die institutionellen (Größe, Finanzen, Recht), und personellen (Werte, politische Ausrichtung) Rahmenbedingungen analysiert. Eine zweite Analysedimension fokussierte auf den Ablauf des Fusionsprozesses. In einem dritten Schritt wurden die Auswirkungen der Fusion auf die Suche nach Behördenmitgliedern, die
Motivation der Mitarbeitenden, die Professionalität der Administration, die Entwicklung der Finanzen und das kirchliche Leben sowie die Angebotssituation untersucht.

\section{Verbreitung von Kirchgemeindefusionen}

Durch Fusionen von Kirchgemeinden hat in der Schweiz die Anzahl Kirchgemeinden im Untersuchungszeitraum zwischen 2010 und 2018 um 8,9 Prozent (absolute Anzahl: 84) bei der reformierten und sieben Prozent (absolute Anzahl: 107) bei der römisch-katholischen Kirche abgenommen, wie Abbildung 2 zeigt.

In der christkatholischen Landeskirche, die in neun Kantonen öffentlich-rechtlich anerkannt ist, gab es Kirchgemeindefusionen lediglich im Kanton Aargau, wo im
Zeitraum von 2010 bis 2018 insgesamt drei Zusammenschlüsse stattfanden und sich die Zahl der Kirchgemeinden von zehn auf sieben reduzierte. In den jüdischen Gemeinden, welche in vier Kantonen anerkannt sind, gab es im Untersuchungszeitrum keine Fusionen und es sind auch keine solchen geplant - einzig eine verstärkte vertragliche Bindung von kleineren Gemeinden an größere. Im Kanton St. Gallen war einmal ein Zusammenschluss zu Beginn der 1950er-Jahre vollzogen worden.

Abbildung 2 berücksichtigt die besonderen kirchlichen Strukturen der reformierten Landeskirche in den Kantonen Bern, Jura und Solothurn. Hier besteht die Kantonalkirche aus Kirchgemeinden dreier Kantone. In den Kantonen Tessin und Wallis werden bei der römisch-katholischen Kirche die Pfarreien nach kanoni- 


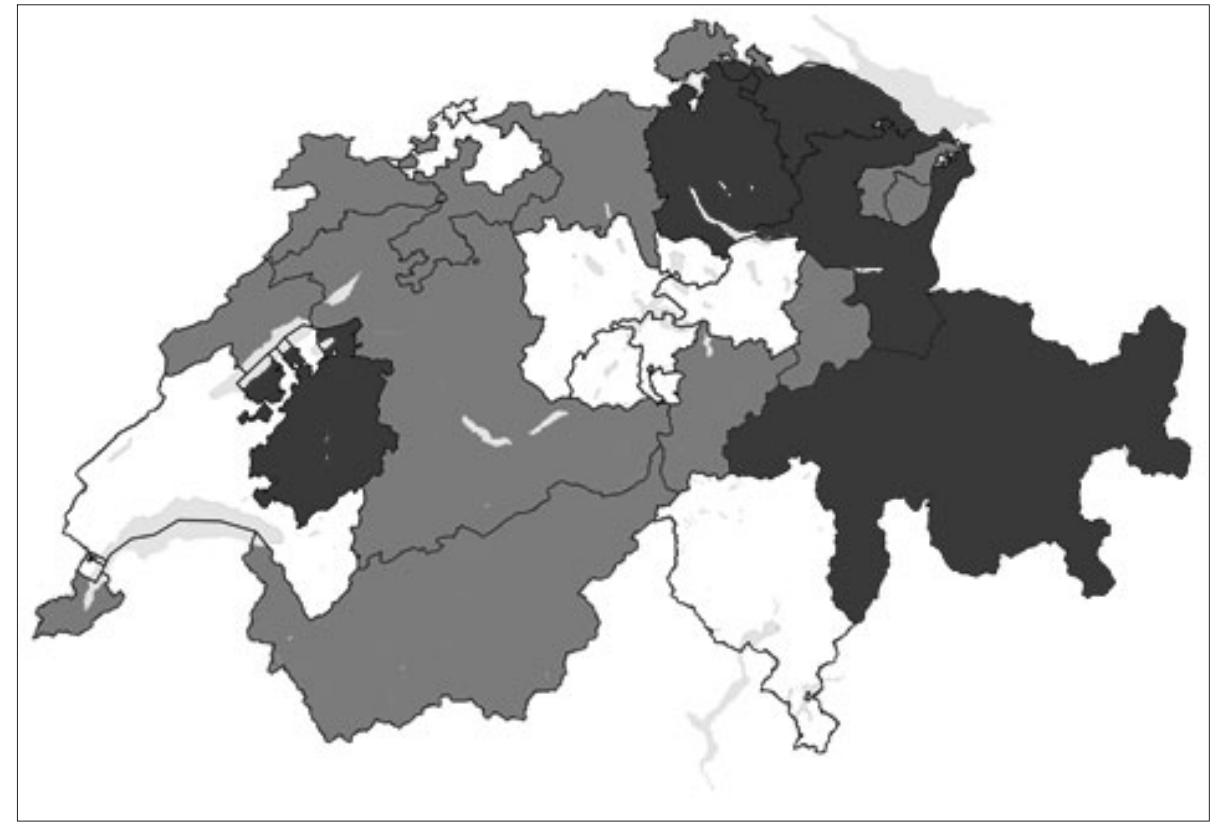

Abb. 3: Intensität der Kirchgemeindefusionen in den Jahren 2010 bis 2018.

Legende: $\mathrm{o}$ Fusionen $=$ weiß, bis 5 Fusionen $=$ hellgrau,$>5$ Fusionen $=$ dunkelgrau

schem Recht aufgeführt, da Kirchgemeinden wie erwähnt als öffentlich-rechtlich anerkannte Institutionen nicht existieren.

Der Vergleich mit der Anzahl Kirchgemeinden pro Kanton ergibt folgende Situation: Fusionen gab es im vergangenen Jahrzehnt bei den beiden großen Konfessionen vor allem in der Ostschweiz und im Kanton Freiburg. Auffällig ist zudem, dass in der Zentralschweiz mit Ausnahme des Kantons Uri, wo die EvangelischReformierte Landeskirche nach der Kirchgemeindefusion noch aus einer einzigen Kirchgemeinde besteht, keine Zusammenschlüsse stattfanden. Gesamthaft gab es in neun Kantonen überhaupt keine Kirchgemeindefusionen, in zwölf ist ein kleiner Rückgang feststellbar und nur in fünf Kantonen kann von einer dynamischen Fusionstätigkeit gesprochen werden, wie Abbildung 3 aufzeigt. In der Regel fusionierten zwei Kirchgemeinden zu einer, es gibt aber auch Fusionen mehrerer Kirchgemeinden, insbesondere im Kanton Graubünden.

In mehr als der Hälfte der Kantone waren Kirchgemeindefusionen per 31.12.2018 ein Thema, wobei beim überwiegenden Teil die Initiative von den Kirchgemeinden selbst ausgeht. 13 Prozent der kantonalen Körperschaften gaben an, dass auch die kantonale Exekutive einen Anstoß dazu gibt. In der Evangelisch-Reformierten Landeskirche des Kan- tons Zürich befindet sich das Thema Fusionen insbesondere aufgrund politischer Vorstöße im kantonalen Kirchenparlament auf der Traktandenliste.

Als Ursache für die realisierten Fusionen spielten aufgrund der Ergebnisse der qualitativen Untersuchung zwar teilweise finanzielle Überlegungen eine Rolle, doch stärker ins Gewicht fielen Schwierigkeiten, genügend Behördenmitglieder zu finden. Als weitere Auslöser von Fusionen wurden spezifische Pfarrsituationen genannt. In Saanen-Gsteig spielte die umfassende Unterstützung durch den Kanton eine große Rolle, sowohl was den finanziellen Beitrag in Form einer Finanzhilfe für die Projektierung und die Umsetzung als auch die Beratungsdienstleistungen betrifft. In Dübendorf-Schwerzenbach kam der Anstoß zum Zusammenschluss vom Reformprojekt der Zürcher Landeskirche. Die Ursachen sind vergleichbar mit den Fusionen von politischen Gemeinden. ${ }^{12}$

20 Kantonalkirchen oder 35 Prozent der Körperschaften bieten eine finanzielle Unterstützung für Fusionsprojekte an. Die verschiedenen Ausgestaltungen von materiellen Beiträgen wie Finanzhilfen für Fusionsabklärungen oder das eigentliche Fusionsprojekt, Übergangsfristen in den Finanzausgleichsbestimmungen oder allgemeine Finanzhilfen (z.B. Entschuldungsbeiträge, Fördergelder) werden von den Kantonalkirchen recht ausgeglichen angewendet. Die evangelisch-reformierte Landeskirche Zürich leistet einen Integrationsbeitrag für das kulturelle Zusammenwachsen der früheren Kirchgemeinden (kulturelle Integration) nach der Fusion. Die katholische Landeskirche Thurgau fängt einen Steuerverlust bei Steuerfußdisparität vor der Fusion auf. Insgesamt zeigt die Auswertung, dass evangelischreformierte Landeskirchen das Instrument finanzieller Unterstützung bei Fusionsabsicht mehr einsetzen als die römischkatholische Kirche. Bei der Unterstützung durch Beratungsdienstleistungen oder andere nicht monetäre Dienstleistungen ist der Anteil an kantonalen Körperschaften, die solche Dienstleistungen anbieten, höher als beim finanziellen Engagement. Nur gerade in zwölf kantonalen Körperschaften erfolgt gar keine Unterstützung.

Nur in den reformierten Landeskirchen der Kantone Glarus und Zürich und den römisch-katholischen Landeskirchen Freiburg und Luzern ist es gemäß Umfrage auch vorgekommen, dass Kirchgemeindefusionen scheiterten.

Regelungen zu Kirchgemeindezusammenschlüssen sind mangels bundesrechtlicher Vorgaben dem kantonalen Staatskirchenrecht zu entnehmen. ${ }^{13} 23$ Kantonalkirchen oder 41 Prozent geben an, über Rechtsgrundlagen für Kirchgemeindefusionen zu verfügen. Dabei wurden die Regelungen meist auf der höchsten rechtlichen Stufe, also auf (Kirchen-) Verfassungsebene, festgeschrieben: in den Organisationsstatuten und Kirchenverfassungen, teilweise in den Kirchgemeindegesetzen. In einigen Kantonen gelten für den Vereinigungsprozess die kantonalen Gesetze (z.B. im Kanton St. Gallen); hier ist lediglich die finanzielle Unterstützung in den kirchlichen Bestimmungen geregelt. Damit kann festgestellt werden, dass unterschiedliche kantonale Regelungen hinsichtlich der Vorgaben des Staates bestehen. Der Kanton Zürich überlässt die Bildung und Organisation der Kirchgemeinden weitgehend den Kirchen. Bern hingegen unterstellt die Kirchgemeinden der kantonalen Gesetzgebung. Der Aargau

12 Steiner/Kaiser 2017, S. $232 \mathrm{ff}$.

13 Friederich 2017, S. 24. 
wiederum legt in seiner Verfassung die Grundsätze fest, ansonsten konstituieren sich die öffentlich-rechtlich anerkannten Landeskirchen selbst.

Auffallend und übereinstimmend betonten die Repräsentanten aller untersuchten Kirchgemeinden die Relevanz der bereits im Vorfeld gepflegten guten Beziehungen zueinander für den Fusionsprozess. Auch die Tatsache, dass die Partnerkirchgemeinden über den gleichen historischen Zugang und einen ähnlichen kulturellen Kontext verfügen, hat scheinbar einen hohen Einfluss sowohl auf das Zustandekommen von Kirchgemeindezusammenschlüssen als auch auf eine nachhaltige Entwicklung in der Folge. Eine gebührende Rücksichtnahme auf den kleineren Fusionspartner war in allen untersuchten Fällen zu beobachten. Überhaupt wurde in den erfolgreich durchgeführten Zusammenschlussprojekten ein hoher lativiert wird diese Feststellung durch das gesellschaftliche Phänomen, dass kirchliche Angebote weniger nachgefragt werden als in der Vergangenheit - daran ändert auch eine Fusion nichts. Auch wenn die verschiedenen seelsorgerlichen Angebote in gleicher Quantität und Qualität angeboten werden, bietet das noch keine Gewähr für eine Trendumkehr, was das Freiwilligen- oder Milizengagement betrifft.

\section{Auswirkungen von Kirchgemeindefusionen}

Die Vermutung, dass dort, wo sich Einwohnergemeinden zusammenschließen, auch Kirchgemeinden fusionieren, kann nicht belegt werden. Die Behauptung ist zwar für die Kantone Freiburg, Graubünden und St. Gallen zutreffend; hingegen gab es beispielsweise im Aargau, wo seit 2010 immerhin acht Prozent der Einwohnergemeinden verschwunden sind, nur

\section{"Zum Fusionserfolg beigetragen hat in den untersuchten Fallstudien eine sichtbare Führung des Prozesses durch die Exekutive der lokalen Kirchgemeinde, eine proaktive Kommunikationskultur und das Ernstnehmen der vorgebrachten Kritikpunkte im Vorfeld der Fusion."}

Stellenwert auf Akzeptanz und Identifikation gelegt. Die Berner Kirchgemeinde, die sich im Alpengebiet befindet, hat - um ein Beispiel zu nennen - einen detaillierten Gottesdienstplan ausgearbeitet, der alle Kirchen und Kapellen berücksichtigt sowie auch Gottesdienste in den Bäuerten (Weiler) vorsieht. Zudem werden auch Berggottesdienste angeboten. Dies führt dazu, dass nach der Fusion mehr Gottesdienste als vorher stattfinden. Die Unterrichtskonzepte für den kirchlichen Unterricht wurden einander angeglichen, auch in Gesprächen mit den Eltern; dies führte nach der Fusion zu einem Mehraufwand im Personalbereich. Gemäß Einschätzung der Interviewten findet die neue Kirchgemeinde Akzeptanz bei den Gläubigen. Re- eine einzige Fusion zweier Kirchgemeinden. Auch in der italienischsprachigen Schweiz (Tessin) fanden im untersuchten Zeitraum keine Zusammenschlüsse von Kirchgemeinden bzw. Pfarreien statt, hingegen ist eine rege Fusionstätigkeit bei den Einwohnergemeinden festzustellen. Wie bei den Einwohnergemeinden der Zentralschweiz fanden mit Ausnahme der Fusion von reformierten Kirchgemeinden im Kanton Uri keine Zusammenschlüsse statt.

Die Vermutung, wonach Zusammenschlüsse innerhalb eines Kantons unter den Religionsgemeinschaften in unterschiedlichen Tempi erfolgen, bestätigt sich. In den Kantonen Bern und Zürich beispielsweise sind es ausschließlich reformierte Kirchgemeinden, die fusionierten. Ausnahmslos römisch-katholische Kirchgemeinden haben sich im Kanton Freiburg vereint. Das mag damit zusammenhängen, dass diese Kantone historisch eine klare konfessionelle Zuordnung besitzen, die sich in verringertem Ausmaß auch heute noch manifestiert, und damit die Kirchenstruktur der dominierenden Konfession von Anfang an feingliedriger war als die Organisation derjenigen Konfession, die erst später und großräumiger entstanden ist. Entsprechend unterschiedlich gestaltet sich der Reformbedarf für Gebietsbereinigungen. Im gemischtkonfessionellen Kanton Graubünden fanden etwa in gleichem Ausmaß Fusionen statt.

Dort, wo die Kantonalkirchen und die Kantone Unterstützung anboten, trugen diese Hilfestellungen zum Fusionserfolg bei. Auch kantonale Anreizprojekte hatten einen Einfluss sowohl auf die Initiierung als auch den Umsetzungsprozess. Ebenfalls ermöglichte eine Zurückhaltung der Kantonalkirchen im Sinne von Druck, dass der Reifungsprozess zum Zusammengehen von Kirchgemeinden weniger konfliktreich war.

Was auf den ersten Blick erstaunlich erscheinen mag, ist die Tatsache, dass alle drei in der qualitativen Untersuchung ausgewählten Kirchgemeinden die Auswirkungen des Zusammenschlusses insgesamt als positiv beurteilen. Erstaunlich deshalb, weil in Diskussionen mit kirchenengagierten Personen Kirchgemeindezusammenschlüsse oft eher kritisch angesehen werden, da befürchtet wird, die Seelsorge werde zentralisiert und nicht mehr in allen Gemeindegebieten angeboten.

Dieses Argument wird jedoch - zumindest bei den untersuchten Fällen - widerlegt, indem Interviews darauf hindeuten, dass Gottesdienste, Religionsunterricht und größere Anlässe in allen ehemals selbstständigen Gemeinden stattfinden. Der Minderheitenschutz wird im kirchlichen Umfeld ernst genommen.

Zwar erfährt das kirchliche Leben nach einer Fusion nicht eine Angebotsausweitung, doch die kirchlichen Dienstleistungen werden auch nicht abgebaut oder zentralisiert, wie zumindest die Beispiele 
in den Kantonen Bern, Aargau und Zürich zeigen. Alle untersuchten Analysedimensionen - Rekrutierung der Behördenmitglieder, Mitarbeitermotivation, Professionalität, Finanzsituation, Entwicklung des kirchlichen Lebens und dezentrale Angebote - werden von den interviewten Personen positiv gesehen; insgesamt gibt es somit Indizien, wonach das kirchliche Leben nach einer Fusion eher gestärkt wird.

\section{Gestaltungsempfehlungen für Kantonalkirchen}

Die Analyse der drei Fallstudien deutet darauf hin, dass es sich für Kantonalkirchen empfiehlt, eine auf die lokalen Gegebenheiten angepasste Fusionspolitik zu betreiben. Dem vorausgehen muss eine
Beitrag einer Kantonalkirche könnte unter dem Aspekt der finanziellen Verlustängste bei den Kirchgemeinden etwa sein, eine Sicherheit bzw. Garantie abzugeben, dass nicht eine „Heiratsstrafe“ - gemeint ist die Kürzung oder der Verlust eines Finanzausgleichsbeitrages - dazu führt, dass Fusionsvorhaben bereits im frühen Stadium scheitern. Um die Akzeptanz einer neuen Kirchgemeinde bei den Gläubigen sicherzustellen, könnte eine Kantonalkirche zudem finanzielle Anreize mit der Forderung verknüpfen, dass ein Grundangebot an kirchlichen Leistungen auch in den einzelnen Gebieten der fusionierten Kirchgemeinde erhalten bleiben muss.

Die mit einer Fusion einhergehende Professionalisierung der Administration schen den potenziellen Fusionspartnern dar. Dieses muss idealerweise bereits vor den ersten Fusionsgesprächen vorhanden sein und gepflegt werden, indem gemeinsame Interessen gesucht und aufgebaut werden. Ein respektvoller Umgang des stärkeren mit dem schwächeren Partner sollte Grundlage sein, was beispielswese in Form von Angebotsgarantien in den einzelnen Quartieren der Gemeinde passieren kann. Eine spezifische Option zum Erhalt der Identifikation steht römischkatholischen Kirchgemeinden offen, indem sie nur auf staatskirchenrechtlicher Ebene fusionieren und die Pfarreien, die kanonisch verfasst sind, eigenständig lassen.

Die Erwartungen zu finanziellen Vorteilen einer Fusion sollten realistisch bleiben: Zwar können Synergien genutzt und die Professionalität der Administration tendenziell verbessert und möglicherweise sogar der Steuerfuß gesenkt werden, doch die Aufrechterhaltung des kirchlichen Lebens und der Unterhalt der Liegenschaften belasten eine fusionierte Kirchgemeinde weiterhin. Denn mit einem Leistungsabbau, der Einsparungen ermöglichen würde, lassen sich Abstimmungen nur schwer gewinnen. Diese Aussage deckt sich mit Studien, die sich mit der Evaluation von umgesetzten Fusionen politischer Gemeinde befassen. ${ }^{15}$

\section{Mehr Fusionen zu erwarten}

der Kantonalkirchen. Neuerungen, wie beispielsweise die Einführung von internationalen Rechnungsstandards (IPSAS) können so umfassender umgesetzt werden. Auch die Besetzung der kommunalen Milizbehörden mit geeigneten und kompetenten Persönlichkeiten ist für die Kantonalkirchen von Relevanz.

\section{Gestaltungsempfehlungen für Kirchgemeinden}

Zum Fusionserfolg beigetragen hat in den untersuchten Fallstudien eine sichtbare Führung des Prozesses durch die Exekutive der lokalen Kirchgemeinde, eine proaktive Kommunikationskultur und das Ernstnehmen der vorgebrachten Kritikpunkte im Vorfeld der Fusion.

Ein entscheidender Faktor für ein $\mathrm{Zu}$ sammengehen stellt das Vertrauen zwi-
Grundsätzlich kann festgestellt werden, dass die Auswirkungen von Fusionen politischer Gemeinden und von Kirchgemeinden vergleichbar sind. Dies trifft auch auf die Ursachen von Fusionen zu, welche bei Kirchgemeinden sogar noch sichtbarer sind als bei politischen Gemeinden. Während die politischen Gemeinden insbesondere an einer sinkenden Partizipation und gelegentlich auch an Finanzproblemen leiden, laufen den staatlich anerkannten christlichen Kirchen in der Schweiz die Mitglieder davon, was zu deutlich sinkenden Steuereinnahmen und einer akzentuierten Schwierigkeit bei der Besetzung der Behördenämter führt. Zu-

14 Steiner/Kaiser/Eythórsson 2016.

15 Steiner/Fetz/Käppeli 2016, S. 894. 
dem werden weniger Leistungen nachgefragt und gleichzeitig muss eine teure Infrastruktur finanziert werden, welche auf die Anzahl an Gläubigen in der Mitte des 20. Jahrhunderts ausgerichtet ist. Von daher ist es eigentlich ein Paradoxon, dass es nach wie vor deutlich mehr Fusionen politischer Gemeinden gibt. Es würde nicht überraschen, wenn sich dies in absehbarer Zeit deutlich ändern würde - auch in den tendenziell strukturkonservativen Kirchgemeinden.

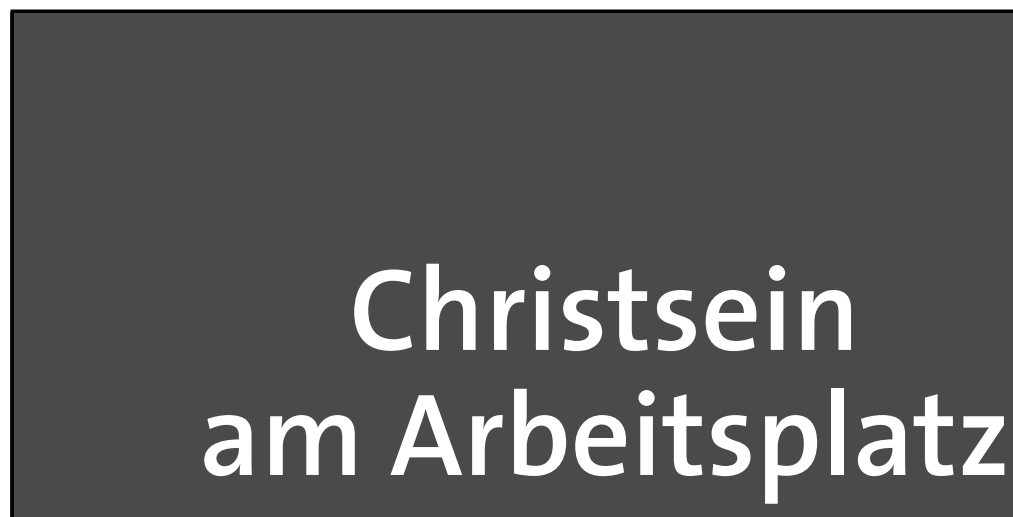

\section{Literatur}

Bundesamt für Statistik (2021): https://www. bfs.admin.ch/bfs/de/home/statistiken/bevoelkerung/sprachen-religionen/religionen.html. Online, abgerufen am 8. Februar 2021.

Burckhardt, J. (1978): Weltgeschichtliche Betrachtungen, Stuttgart.

Friederich, U. (2017): Rechtliche und praktische Fragen zum Zusammenschluss von Kirchgemeinden. Schweizerisches Jahrbuch für Kirchenrecht, Bern.

Kley, A. (2001): Das Religionsrecht der alten und neuen Bundesverfassung ( $R$. Pahud de Mortanges, Hrsg.), Fribourg.

Kosch, D. (2007): Demokratisch, solidarisch, unternehmerisch: Organisation, Finanzierung und Management in der katholischen Kirche in der Schweiz, Zürich.

Kosch, D. (2020): Staat und Religion in der Schweiz des 21. Jahrhunderts: Kirchenreform im Kontext des dualen Systems, Zürich.

Kunz, R./Schlag, T. (2017): Gemeindeautonomie und Zuordnungsmodell in reformierter Perspektive. Kirchentheoretische Orientierungen und Folgerungen für die kirchenleitende Praxis. Schweizerisches Jahrbuch für Kirchenrecht, Bern.

Stadler, H. (2010): Mittelalterliche Wurzeln des Schweizer Staatskirchenrechts, Zürich, SKZ 4142.

Steiner, R./Kaiser, C. (2017): Effects of amalgamations: evidence from Swiss municipalities. In: Public Management Review, 19. Jg., Nr. 2, S. 232252.

Steiner, R./Fetz, U./Käppeli, S. (2016): Gestaltung von Gemeindefusionsprozessen. In: Praxishandbuch Public Management, hrsg. v. Andreas Bergmann et al., Zürich, S. 880-896.

Steiner, R./Kaiser, C./Eythórsson, G.T. (2016). A Comparative Analysis of Amalgamation Reforms in Selected European Countries. In Local Public Sector Reforms in Times of Crisis, London.

Von Campenhausen, A./De Wall, H. (2006). Staatskirchenrecht: eine systematische Darstellung des Religionsverfassungsrechts in Deutschland und Europa; ein Studienbuch, München.

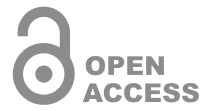

The Christian Body at Work
Spirituality, Embodiment,
and Christian Living
Von Dr. Tobias Brügger
2021, 375 S., brosch., 59,- $€$
ISBN 978-3-8487-7853-9
(Religion - Wirtschaft - Politik, Bd. 21)
In englischer Sprache

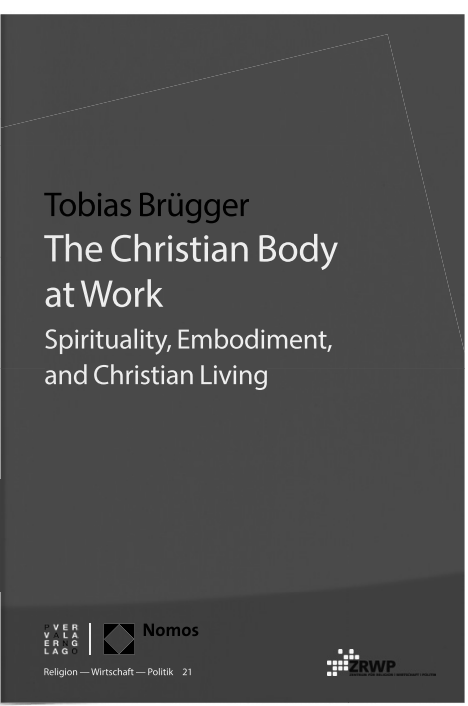

Ein Großteil der arbeitenden Bevölkerung weltweit gilt als christlich affiliiert, doch was bedeutet Christsein am Arbeitsplatz? Die Studie beleuchtet dazu Forschung aus Management- und Organisationswissenschaften und Theologie, präsentiert Feldforschung zu ManagerInnen in der Schweiz und entwickelt eine Verkörperungsperspektive. Der Autor benennt Schwierigkeiten der vorherrschenden Verwendung von Begriffen wie Glaube, Spiritualität oder Religion am Arbeitsplatz und plädiert dafür, Framing-Praktiken und die Körperhaftigkeit christlicher Existenz zu berücksichtigen. Indem ChristInnen in körperlicher Form an Christi Tod und Auferstehung teilhaben und so Christus verkörpern, finden sie sich an einer existentiellen Schaltstelle für die Gestaltung von Arbeitsaktivitäten. Die Studie zeigt, wie der Fokus auf „Christinnen und Christen“ einen Raum für relevante organisations- und managementbezogene, soziologische, ethische und theologische Aspekte zeitgenössischer Arbeitskontexte eröffnet.

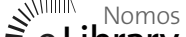 \\ હ e Library nomos-elibrary.de}

Bestellen Sie jetzt telefonisch unter +49 7221 2104-37 Portofreie Buch-Bestellungen unter nomos-shop.de Alle Preise inkl. Mehrwertsteuer 